resulted in the stretching, displacing, breaking, and cutting of the nerves. I therefore took an old nerve scalpel and blunted its cutting edge and point, and employed it as a needle for teasing out the fat, cellular tissue, \&c., in which the nerves were imbedded. The fat, being partially melted by the hot water, was in the best possible condition for being teased out and, when so treated, it floated away. I never employed a knife and very rarely scissors. By these means I was enabled to dissect the most delicate cardiac nerves in situ. They were in no case dragged or displaced. The hot water, moreover, always kept them taut and as they appeared prior to dissection.

The hot-water method of dissecting was, in warm weather, very oppressive and severe on the eyes, but very satisfactory. The more heat and light, the better the result. It had only one drawback : the hearts if not worked off in three or four days at most were apt to become soft and to putrefy. 'This tendency to decay involved continuous work and a great strain while dissecting, so much so that, looking back, I am inclined to believe that the hot-water nerve-dissections were the most troublesome and difficult I have ever executed. When the hearts which were dissected under hot water were freed from fat, cellular tissue, \&c., and the nerves were carefully dissected out, I boiled them, in some cases, in sulphuric ether-an expensive and ticklish process-resulting, in my case, in two rather serious explosions. The hot ether dissolved any tiny particles of fat which had escaped the hot water and my improvised blunted teasing scalpel. The nerve-dissections of the heart prepared in this way presented such a clean smooth surface that in some cases they appeared more or less polished. One great advantage of the process was the non-stretching and keeping of the nerves in exactly their original positions; the hot water, as explained, preventing the nerves from becoming lax. Care had to be exercised as to the temperature of the water employed; if too hot it shrivelled the nerves, if too cold the fat was not melted and could not be teased out.

As it was necessary in certain cases to distinguish the nerves from the finer blood-vessels, capillaries, and lymphatics I resorted in not a few instances to injecting the cardiac blood-vessels. I explained to Mr. Stirling, who worked in an adjoining room, that $\mathrm{I}$ would require to employ an injection which could be forced into the blood-ressels in the cold state and which would stand the heat and not shrivel on cooling. He at once suggested a cold injection of flour-and-water coloured with vermilion for the arteries and ultramarine blue for the veins. The idea was to make a stiff paste within the vessels by means of the hot water. This homely injection suited my purpose admirably and nearly all of my injected nerve-dissections of the heart were so treated. The nerves of the heart, as already indicated, were much more difficult to dissect than the muscular fibres of the ventricles. They were gossamer in texture and the slightest slip of even the blunted scalpel made havoc. It was a case of constant watching and the strain on head, eyes, and hand was very trying. I worked at the nerve-dissections from $8 \mathrm{~A}$, M. to 6 P.M. each day for a whole summer and autumn with an interval of an hour for luncheon. Nine hours' continuous work in and over hot water and in the heat and glare of summer and autumn was, to say the least, not a little fatiguing. Experience, however, taught me that it was necessary to finish the nerve-dissections with all possible dispatch. There was, as stated, a danger of their softening, and even decomposing, if the dissection was too long continued. The hot water and the heat of summer necessarily emphasised the danger. I was, however, between Scylla and Charybdis. A strong light and a strong heat were both necessary to enable me to accomplish the delicate work on which I was engaged.

The summer session of 1860 was one of the busiest of my life. I got up each morning at four o'clock, and, in the early hours wrote an essay on the "Presumption of Survivorship" which secured for me the gold medal in the class of medical jurisprudence. This essay was published in the British and Foreign Medico-Chirurgical Rcview for January, 1865. The nerve-dissections of the heart, as it turned out, were wholly successful, and, with my inaugural dissertation describing them, obtained for me in 1861, when I graduated in medicine, a thesis gold medal, the highest honour the University of Edinburgh confers. The dissertation, which was illustrated and contained drawings of the nerves of the heart and of the microscopic appearances presented by the ganglia of the nerves, was deposited in the University of Edinburgh Library where it may be consulted. The nervedissections themselyes I presented to the anatomical museum of my alma mater where they can be examined. I mounted them in glass jars with glass lids as I had done the muscularfibre preparations of the ventricles. The muscular fibre and nerve-dissections of the heart presented by me to the Anatomical Museum of the University of Edinburgh number in all 164. I subsequently photographed the nerve-dissections as I had done the muscular fibre ones, and a short account of them appeared in the proceedings of the Royal Society of Edinburgh for 1865 . They were also described and figured in my lectures "On the Physiology of the Circulation in Plants, in the Lower Animals, and in Man," which were originally published in the Edinburgh Medicat Journal during the years 1872 and 1873 , and subsequently republished by Messrs. Macmillan in book form in England and America in 1874, with 150 illustrations on wood.

(To be continued.)

\section{A NOTE ON NEISSER'S TEST FOR DIPHTHERIA BACILLI.}

\section{Bx L. COBBETT, M.A., M.D. CANTAB., F.R.O.S. ENG., L. R.O.P. LoND. \\ (From the Pathological Laboratory, Cambridge.)}

Among the facts which have in recent years come to light about the etiology of diphtheria two stand out as being of special practical importance ${ }^{1}$; I refer to the occurrence of virulent and dangerous diphtheria bacilli in the mouths of certain healthy persons, and to the fact that these persons are to be found only among those who have come into contact with the sick or with others who, like themselves, harbour the bacilli. These facts make it probable, and direct evidence is not wanting to show, that diphtheria is spread by those who, having come into contact with the sick, acquire the bacilli without being themselves ill, and who, not being recognised as infectious, are often allowed to move about freely, and are even sent to school, as much as, if not more than, by those who fall ill of the disease and are for the most part removed to hospital or isolated at home.

The duty of discovering, isolating, and disinfecting the former class of persons is becoming more and more the urgent duty of sanitary authorities. For the fact that they are not scattered broadcast throughout the community, as was once supposed, but are confined to the class of persons whom we conveniently call " contacts," renders their discovery a practical possibility and offers a fair prospect that at least the great majority of them may in the near future be subjected to isolation and antiseptic treatment, with immense advantage to the public health. The systematic use of this method of prevention in Cambridge and Colchester has, however, involved a great amount of work. Therefore anything which simplifies the methods of examination and renders them more certain will materially help to bring about the general adoption of this preventive measure by our sanitary authorities. Since the beginning of the year in Cambridge 900, and in Colchester since August about 1500 , cultures have been examined for diphtheria bacilli. These include many from patients and convalescents, but the majority were from healthy contacts, mostly children.

The examination of cultures from healthy persons is apt to be a more arduous and delicate business than the examination of those from patients and convalescents, and often far more depends on an accurate report. It is particularly the case in dealing with private schools that one must be on one's guard against reporting as diphtheria bacilli the longer pseudo-diphtheria forms of Hofmann's bacillus. Moreover, when diphtheria bacilli are present they are often relatively few and are apt to be hidden in a crowd of other forms and therefore difficult to discover. In making the microscopic examinations here referred to the aim has been to make, as far as was possible, each single preparation from a single colony. Under these circumstances bacteria are more easily recognised than when different kinds are mixed together.

1 I do not here refer to the important fact that diphtheria bacilli persist in the mouths of some convalescents long after the period when they were formerly considered free from infection, because it is already well recognised. It is, however, not so generally recognised that evidence of the complete disappearance of the bacilli cannot be conexaminations. 
This is particularly the case when there are a few colonies of diphtheria bacilli among a lot of Hofmann bacilli. When a little group of the former is mixed with a lot of the latter it is difficult to say whether they are a group of the long or giant forms of Hofmann's bacillus or whether they are true diphtheria bacilli. But if a preparation can be made from a single colony which is thus shown to be composed entirely of the long bacilli the difficulty disappears. Several such preparations are made on a single cover-slip and arranged in parallel lines, in order that a considerable number of colonies may be passed in review in a short time. The preparations are stained and mounted in dilute methylene blue (1 in 5).

In all cases of doubt, and in most cases where bacilli bearing even a remote resemblance to the diphtheria or pseudo-diphtheria bacilli were seen, Neisser's stain has also been used. Without its aid diphtheria bacilli would have been undoubtedly missed on several occasions. This occurred when the diphtheria bacilli were mixed with a crowd of Hofmann bacilli, the character of the culture not admitting of the satisfactory examination of single colonies. In these cases Neisser's stain first revealed the diphtheria bacilli, and their subsequent isolation and examination of pure cultures showed that the stain had given the right indication. In such cases when the colonies of diphtheria bacilli are few in number and crowded with other sorts it is often difficult and tedious to prepare a second cover-slip which shall contain the bacilli which one wishes to subject to Neisser's stain. Hence arises the necessity for applying the special stain to the original cover-slip. This may be floated off, after removing the cedar oil, with filter-paper soaked in xylol, washed for a minute in 5 per cent. acetic acid, and stained by the ordinary Neisser method.

But frequently one desires to apply the test to a particular group of bacilli without moving the slide from the microscope. This led me to try the device of applying a drop of 5 per cent. acetic acid to one edge of the cover-slip and drawing the fluid under the glass by means of a small piece of filter-paper placed on the other side. If the bacilli are watched while the acid is entering under the cover-slip one sees first a current of fluid sweeping up loose bacilli and hurrying them away. A blue cloud next appears and blots out everything for a second and passes on. 'Then once again the field is bright and clear, and the diphtheria bacilli, if such they are, show the characteristic polar bodies as if stained in the way Neisser recommended; only the bodies of the bacilli are not brown, but pale-blue. Hofmann's bacilli also have a fairly characteristic appearance when treated in this way. They do not so easily decolourise as the diphtheria bacillus and at about the middle of each half a good deal of blue usually remains. With diphtheria bacilli the change is instantaneous, and the picture revealed is often, I think, not inferior to that of a Neisser specimen stained in the usual way. The method is so quick that it takes no appreciable time and can therefore be applied, without causing delay, to every preparation which contains micro-organisms which have the remotest resemblance to the diphtheria bacilli. I now constantly use it. For a long time I was in the habit of controlling the acid test by staining another preparation by the usual Neisser method. The results showed that when typical polar bodies are disclosed by the acid there is no necessity to use the full Neisser method. The utility of the latter seems to be restricted to the cases where the acid method gives an indecisive result. But it must be added that when the acid test is unsatisfactory the orthodox Niesser stain does not give a much better result.

I have already expressed my great appreciation of Neisser's method as a means of distinguishing the diphtheria from the pseudo-diphtheria bacillus, but it is not infallible. More than once a diphtheria bacillus (subsequently proved to be an acid-former and to be virulent for guinea-pigs) has been found, which showed no polar bodies when stained with the usual Neisser method. ${ }^{2}$ And more frequently cultures of diphtheria bacilli have been found which showed but few polar bodies and those very small and difficult to find. In such cases I find little or no advantage in staining for two minutes in the acid blue as has recently been recommended. On the other hand, when Hofmann's bacilli are treated with Neisser's stain (or with acetic acid after methylene blue) I have several times seen a few bacilli which showed very minute polar bodies. These cultures had been sown on the previous day and were therefore about 24 hours old

2 In sub-cultures they were either poorly developed or absent. or less. Such bacilli have been isolated and investigated with the result that they proved to be Hofmann bacilli.

Neisser's stain, therefore, fails to show polar bodies in a small proportion of true diphtheria bacilli. And it shows minute and doubtful polar bodies in a few Hofmann bacilli. This fact detracts very little from the value of the stain as a differential test, because the exceptions to the general rule are so few. We must, then, hold that a good positive reaction is positive evidence that the bacilli are diphtheria bacilli, that a definite negative reaction is valuable evidence, but not alone conclusive, while a poor or doubtful reaction is not of much value either way.

In conclusion, I feel justified in recommending the acetic acid modification of Neisser's stain which I have described to the notice of others engaged in this work.

Cambridge.

\section{SOME PRACTICAL POINTS IN THE TREAT- MENT OF CASES OF FRACTURED \\ PELVIS WITH RUPTURED BLADDER AND OF CASES OF RUPTURED URETHRA.}

BY C. J. BOND, F.R.C.S. ENG.,

SURGEON TO THE LETOESTER INFIRMARY.

THERE is a certain kind of accident which seems especially characteristic of the hunting field, in which the horse rolls on the prostrate rider, crushing the pelvis and causing injuries which frequently involve the bladder or the urethra, or both. Since the report of cases by me in THE LANCET of August 10th, 1889, p. 260 , describing a method of draining the injured bladder by a combined supra- and infra-pubic route, on a special staff, several such injuries have come under my notice. In all these the condition of the patient after injury is much alike. There is great pain on any movement disturbing the fractured bones, while if the thighs are gently raised they can be flexed slightly on the pelvis without undue pain. There is the tender, board-like, dull area above the pubes, spreading laterally to the groins, which is due to the extravasation of blood, and in the case of injury to the bladder the addition of extravasated urine in the pre-vesical space. There is inability to empty the bladder, and if on gently attempting to pass a catheter obstruction be encountered close to the neck of the bladder, and especially if blood be drawn and the bladder cannot be reached, then in all probability injury of the urethra also exists.

There are one or two points about the combination of fractured pelvis with injury to the bladder and urethra which are worth notice.

In all my cases of fractured pelvis in which the bladder has been torn the rent has been extra-peritoneal, and in the anterior wall of the bladder, and in my opinion it is caused by the direct puncturing of the vesical wall by the fractured bones. This is so constant an association that the pre-vesical space should be opened first in all these cases of bladder injury, the presence of blood and urine will then reveal the nature of the injury, and the peritoneum need not be opened, unless injured, as the posterior wall of the bladder can be explored by the finger from within the viscus.

With regard to the urethra, the position of the patient's body in relation to the crnsh seems to be the deciding factor. In my experience the urethra gets injured where, after the fall, the crush comes with the patient lying on his side, thus driving the fractured sides of the pubic arch together like the blades of a pair of scissors and injuring or completely dividing the urethra in its membranous portion, just in front of the apex of the prostate. On the other hand, where the pelvis is crushed from before back the urethra is more likely to escape. In the commoner example of ruptured urethra, the result of a fall astride some beam or other obstacle, the injury is the result of the crushing of the soft urethra against the unyielding bones of the pubic arch, and the anterior or distal portion is torn away from the more fixed posterior or proximal end. In this accident the rupture generally occurs rather further forward than in fractured pelvis, and the separation of the torn ends may be considerable-as much as one or two inches. 